\title{
Impact of Transient Response of Erbium-Doped Fiber Amplifier for OPS/WDM and its Mitigation
}

\author{
Yoshinari Awaji ${ }^{1}$, Hideaki Furukawa ${ }^{1}$, Naoya Wada ${ }^{1}$, Eddie Kong $^{2}$, Peter Chan ${ }^{2}$, \\ Ray $\mathrm{Man}^{2}$ \\ ${ }^{1}$ National Institute of Information and Communications Technology (NICT), 4-2-1, \\ Nukuikita, Koganei, Tokyo 184-8795, Japan \\ \{yossy, furukawa, wada\}@nict.go.jp \\ ${ }^{2}$ Amonics Ltd., Unit 101, 1/F, Winning Centre, 29 Tai Yau Street, San Po Kong, Kowloon, \\ Hong Kong \\ \{eddie, peter, $\underline{\operatorname{rayman}\} @ \text { amonics.com }}$
}

\begin{abstract}
We investigated impairments of optical packets when these packets amplified by cascaded EDFAs in several distinctive cases in WDM environment. The origin of impairments were mainly gain excursion and additional noise come from inter-channel cross talk between WDM channels, and the degree of impairment changed according to link utilization. Moreover, the impairment is cumulative by increase of the number of EDFAs cascaded. It means that the tolerance for error of optical packet receiver substantially reduces according to the traffic intensity and the number of hops each packet experiences. We proposed the mitigation by adopting special class of EDF which have larger active erbium core. Our methodology can supplement conventional electrical controlling measures against transient responses which have insufficient response speed in the application for short optical packet of sub-micro seconds long.
\end{abstract}

Keywords: EDFA, Gain excursion, Cross gain saturation, Optical packet, WDM

\section{Introduction}

Development of optical packet transporting stratum is a fundamental issue to assure transparency, granularity, high efficiency of bandwidth utilization, agility of forwarding, and of course, high wire rate in optical telecommunications. Indeed, continuous bit stream or flame train with fixed repetition are easy to be transmitted on optical domain and to be operated by simple architecture in control plane, however, it can promise low efficiency on physical layer. Such kind waste of given resource is inherently irretrievable by effort in network architecture. Sophisticated techniques to handle optical packets are indispensable.

Optical packet carries not only messages of users but also functional signals upon requests of control plane. Therefore, the length and frequency of optical packet are various. As well known, such bursty intensity-modulated signals cause dynamic 
transient of envelope of packet when it is optically amplified by erbium-doped fiber amplifiers (EDFAs) [1].

As a matter of fact, similar distortion of envelope matters in electronic amplifiers mainly because of capacitance and drift of DC balance. Therefore, several artifices, such as finely tuned additional capacitance, electronic gain controlling, coding, and so on, were necessary to realize workable physical layer to handle electric packets.

The dynamic transient in EDFA has another origin. It is caused by consumption of excited population of $\mathrm{Er}^{3+}$ ions in gain media as a result of amplification itself and cross gain saturation in wavelength division multiplexing (WDM) environment [2]. Therefore, the transient in EDFA is inherently unavoidable as far as using intensitymodulated optical packets.

The measures previously reported are roughly classified to two categories, one is electronic approaches [3] including the automatic gain controlling (AGC) and another is optical approaches [4] including optical feedback loop. The electronic approaches suppose that the natural lifetime of $\mathrm{Er}^{3+}$ ions is around $10 \mathrm{~ms}$ which define the gain dynamics of EDFA. On the other hand, the AGC can respond within sub-micro second [3]. It seems to be enough, but if we assume 10Gbps and 500bytes (typical average size on the Internet backbone) packet, the duration of the packet is only 400ns and the AGC cannot treat such short packet. Perhaps the transient in such relatively short optical packet had ignored comparing the natural lifetime. However, it is not negligible [5].

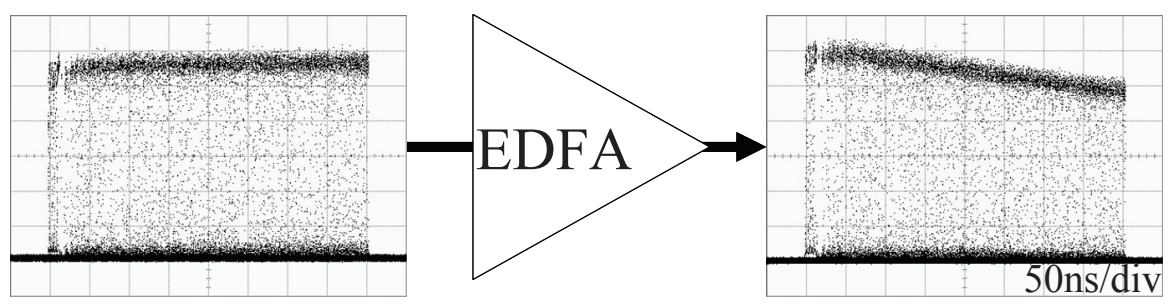

Fig. 1. An example of transient in optical packet envelope cause by EDFA. A packet consists of 128 bits preamble and 3814 bits payload at $9.95328 \mathrm{Gbps}$, then the duration of packet was about 400ns.

We have observed the dynamic transient of EDFA for 400ns packet in single wavelength [5]. Because of short duration, it did not seem as optical surge but gradual gain excursion shown in Fig. 1. The degree of transient might be cumulative [1], and it is clear that such transient can seriously affect clock and data recovery (CDR) on receiver. Moreover, it is very difficult to be compensated except 2-R methodology using optical-electrical-optical (OEO) conversion which makes the optical forwarding opaque and limits available bandwidth.

On the other hand, optical controlling approaches resulted as insufficient suppression of transient or needed unfavorable complexity.

As one of effective mitigation, we have suggested the adoption of new class of EDF which can be applied in parallel with existing method to control EDFA [5]. 
In this paper, we observed the properties of transient response of cascaded EDFAs with optical packet over WDM. We confirmed that our proposed mitigation presented the best result within available devices under test (DUTs) even in WDM environment, hence the validity of our approach was proved. Finally, we discuss the impact of transient for optical packet switch (OPS) with WDM. We respectfully call attention about the gain excursion issue in designing of EDFA dedicated to OPS with WDM.

\section{Concept of Examination}

\subsection{Formula of gain excursion}

The gain excursion of EDFA can be approximated by the following formula [2].

$$
\begin{gathered}
G^{\prime}(0)=\frac{[G(\infty)-G(0)]}{\tau_{0}}\left[1+\sum_{j} \frac{P^{\text {out }}\left(\lambda_{j}\right)}{P^{I S}\left(\lambda_{j}\right)}\right] \\
P^{I S}\left(\lambda_{j}\right)=\frac{h v S}{\left[\sigma_{a}\left(\lambda_{j}\right)+\sigma_{e}\left(\lambda_{j}\right)\right] \Gamma_{j} \tau_{0}}
\end{gathered}
$$

$G(0)$ is the gain before the transient and $G(\infty)$ is the steady gain after the transient, respectively. $\tau_{0}$ is the intrinsic lifetime of the upper level of the erbium ions (We assumed two level systems of $\mathrm{Er}^{3+}$ even for three level pumping [5]). $P^{I S}$ is the intrinsic saturation power at the wavelength channels. $S$ is the active erbium area of EDF, $\sigma_{a}$ and $\sigma_{e}$ are the absorption and stimulated emission cross section at the wavelength channels, $\Gamma$ is the confinement factor which is the overlap integral between the $\mathrm{Er}^{3+}$ ions and the mode field of light, respectively. In this formula, signal and pump light can be treated identically [6].

The $G^{\prime}(0)$ is the initial slope of gain excursion and is inverse proportional to $S$. An improvement on gain excursion should be made by using EDF with larger active erbium area. We compared a two types of commercially available EDFAs (called as Type A and B) with our prototype of EDFA (called as Type C) optimized for OPS adopting an EDF with enhanced active area whose diameter was $4.3 \mu \mathrm{m}$.

\subsection{Form of optical packet}

We have developed optical packet transmitter which generated WDM packet stream segmented to eight wavelengths from 10G-Ether flame input [7,8]. Fig.2 shows packet format and traffic intensity in demonstration. Bit rate of payload conformed to OC-192 (9.95328Gbps) and a packet consists of 128bits preamble and 3814bits payload, then the duration of a packet was about 400ns. We compare two different 
link utilization (LU [\%] = summarized total duration of packets within a second) of $33 \%$ and $0.4 \%$.

Comparing the actual experimental setup, the formula (1-2) present only approximative understanding because the extinction ratio of NRZ modulated signal was not ideal and typically $10 \mathrm{~dB}$. Hence, there is residual $\mathrm{cw}$ light and amplified spontaneous emission (ASE). For example, the summation of optical energies forming mark (" 1 ") bits are only $1 / 50$ of total optical energy in the case of $\mathrm{LU}=0.4 \%$, taking into account that average mark ratio of packet was 50:50. The rest of optical energy belongs to DC component on space ("0") bits or blank time between packets. The energy for effective optical pulse is not dominant. On the other hands, the formula assumed fast switched WDM networks in which the bit stream is continuous, and pumping power for EDF was successfully consumed by effective optical pulses and explicit ASE component. However, our experience qualitatively agreed with the formula up to now. The precise and quantitative examination of formula should be future issue.

\section{Packet format}

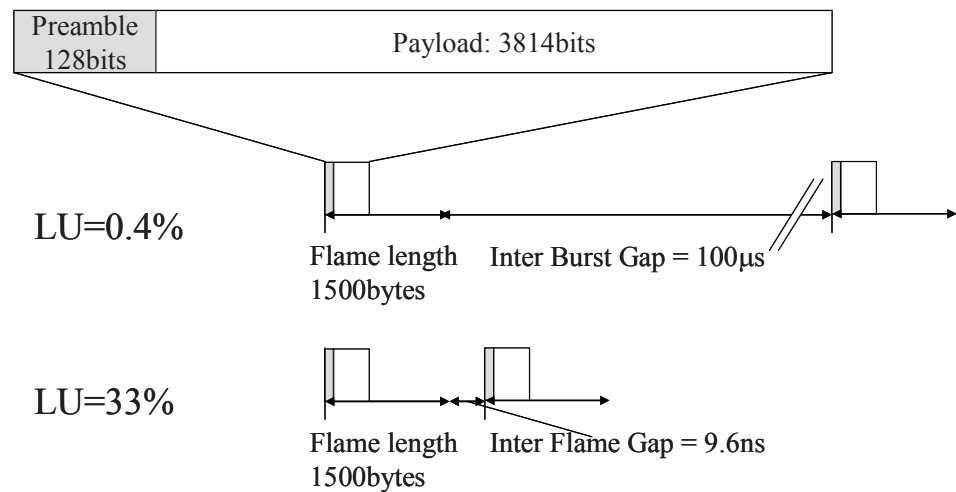

Fig. 2. Packet format and two different traffic intensities. LU: Link Utilization (summarized total duration of packets within a second)

Fig. 3 shows wavelength and timing allocations of packets in several distinctive cases in WDM environment. The packet transmitter originally generates $100 \mathrm{GHz}$ spaced eight wavelengths of packet at same timing (Pattern I). We also observed the decorrelated WDM packet stream (Pattern II) for comparison. The choice of Pattern I and Pattern II should depend on the network architecture, and these are typical case from the point of view of effect of cross gain saturation in EDFAs resulting in transient response.

Reminding that the formula also indicates that optical power on other wavelength affect the gain excursion (cross gain saturation), it should be more serious in WDM than our previous report in single wavelength [5]. To emphasize it we also observed Pattern III and IV. 

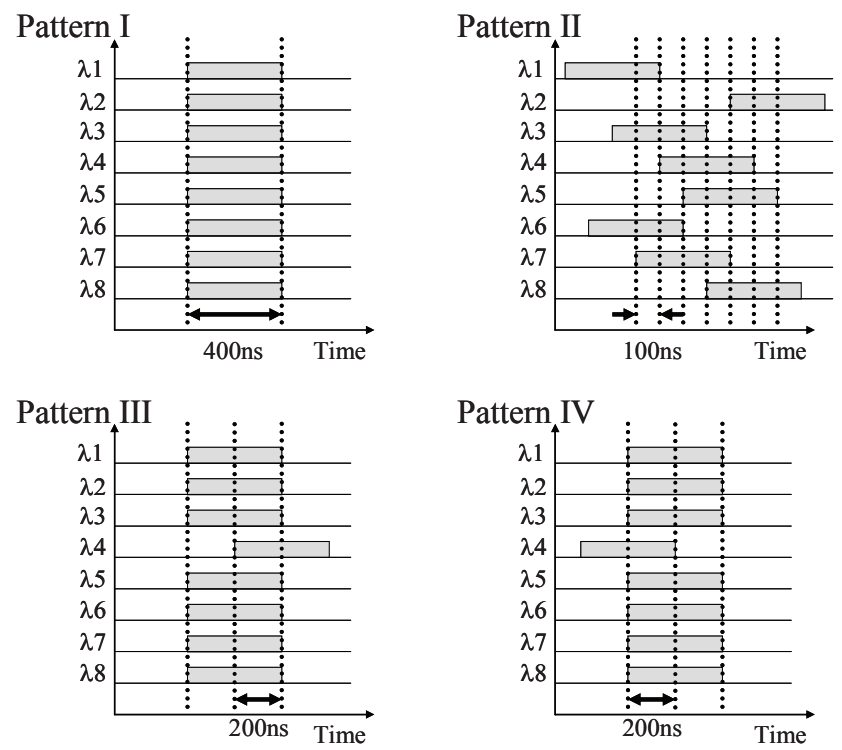

Fig. 3. Wavelength and timing allocations of packets

\subsection{Experiment}

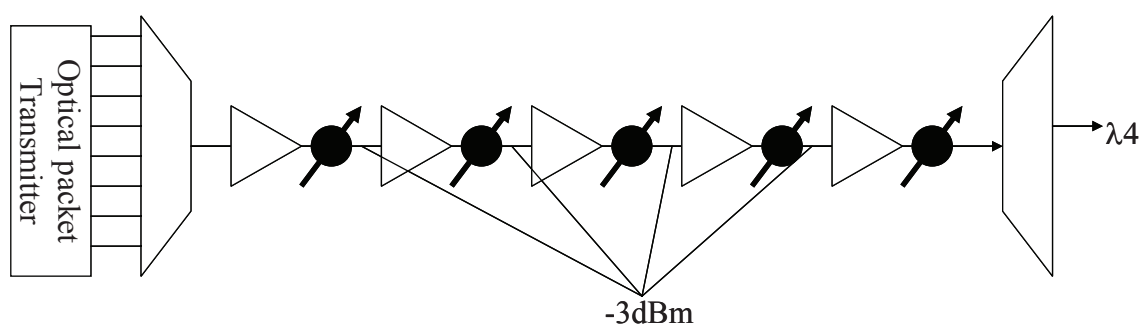

Fig. 4. Schematic drawing of experimental setup. Black circle with arrow indicates variable attenuator assuming optical losses derived from optical components.

Fig.4 shows schematic drawing of experimental setup. We assumed the model of setup as a typical configuration of OPS and cascaded up to five EDFAs. It can also be applicable to the model of optical packet network with several hops. The initial average input power into the first EDFA was $-8 \sim-12 \mathrm{dBm}$ depend on the LU and it was amplified up to $+13 \mathrm{dBm}$. Then it was filtered with $7.6 \mathrm{~nm}$ band pass filter and attenuated to $-3 \mathrm{dBm}$ and injected into latter EDFA. Each gain of EDFAs was adjusted to $16 \mathrm{~dB}$. We observed $\lambda 4(1550.12 \mathrm{~nm})$ only. 
Table 1. Combination of condition

\begin{tabular}{|l|l|c|}
\hline Type of EDFA & LU(Link Utilization)[\%] & Wavelength and timing allocation \\
\hline $\begin{array}{l}\text { A } \\
\text { (Commercial) }\end{array}$ & $33 \%$ & I \\
\hline B (Commercial) & $0.4 \%$ & II \\
\hline C (Proposed) & & III \\
\hline & & IV \\
\hline
\end{tabular}

\section{$3 \quad$ Results}

At first, we summarized the result of observation.

As a comparison of the type of EDFA, Type A is the worst and Type $\mathrm{C}$ is the best. Type B is similar to Type C but slghtly worse.

As a comparison of $\mathrm{LU}, \mathrm{LU}=33 \%$ present no significant impairment in almost case except Type A. We observed slight additional noise for Type A. To the contrary, $\mathrm{LU}=0.4 \%$ condition was severe and many impairment were observed.

It was also confirmed that the the degree of impairment was cumulative depending on the number of EDFAs cascaded by comparing with single stage of EDFA.

And we show several distinctive results according to wavelength and timing allocations in Fig. 5.

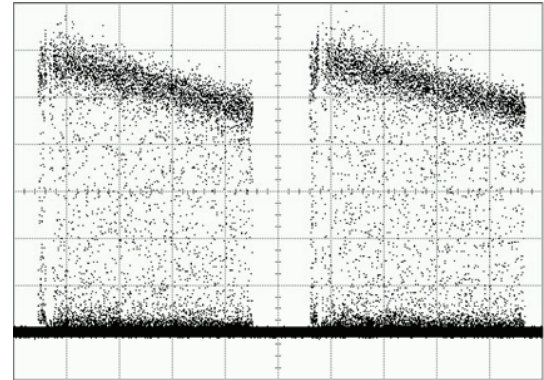

(a)

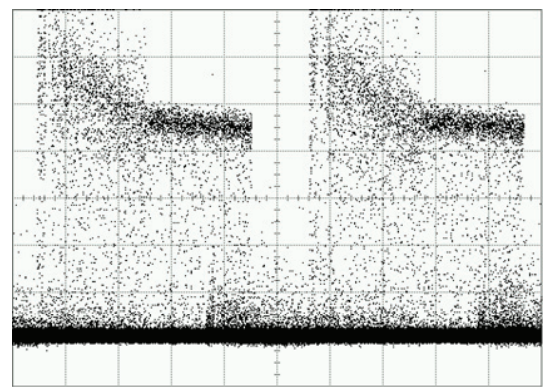

(c)

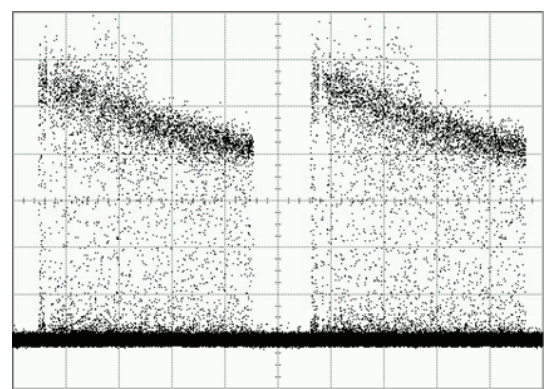

(b)

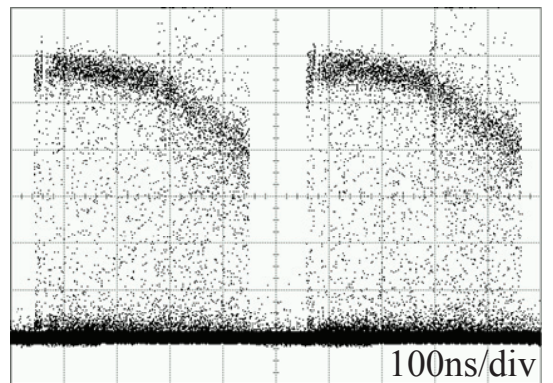

(d)

Fig. 5. Impairment derived from gain excursion and cross talk (caused by cross gain saturation). LU=0.4\%. (a) Pattern I, Type B, (b) Pattern II, Type A, (c) Pattern III, Type A, (d) Pattern IV, Type A 
Fig. 5 (a) shows that optical packet suffer gain excursion as a result of five cascades of EDFAs. Such degree of impairment was unavoidable in present. Type B tends to relatively low excursion but accumulation of excursions occerred even all wavelength have same timing.

Fig.(b) (d) show the worst case with Type A. (b) There is additional bursty noise around the time when density of de-correlated packets on other wavelengths is high. It is clearly shown in (c) and (d) that seven channels other than observed one seriously affect on $\lambda 4$ when they are dropped or added. To emphasize the impairment, the timing of seven channels are same. It points out that usage of unoptimized EDFA in WDM environment eventually result in such destruction of optical packet.

We observed the best result in all cases with Type $\mathrm{C}$ adopting proposed mitigation which is larger erbium core. Fig.6 shows the observed result with Type C corresponding to Fig.5.

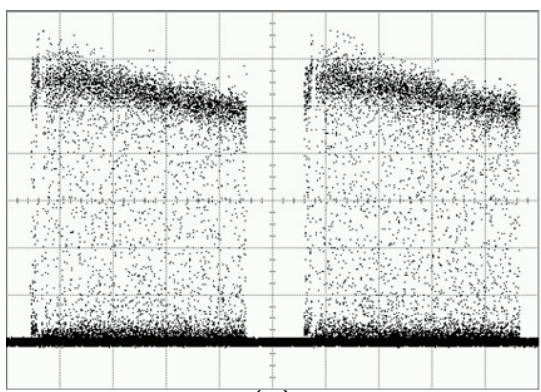

(a)

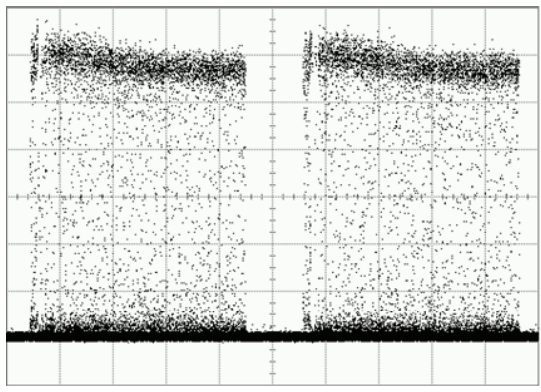

(c)

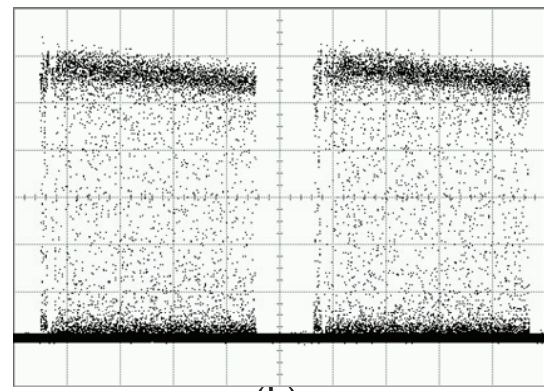

(b)

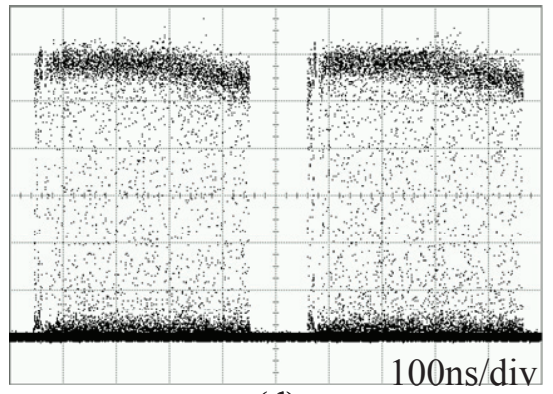

(d)

Fig. 6. The best result with Type $\mathrm{C}$ adopting proposed mitigation corresponding Fig.5. LU $=0.4 \%$. (a) Pattern I, (b) Pattern II, (c) Pattern III, (d) Pattern IV

Fig.6 (a) is almost same with Fig.5 (a) but 30\% improved. Moreover, (b) (d) show slight excursion but no significant additional noise come from cross talk while Type A shows heavy impairement. It is clear that our approach certainly mitigate gain excursion and cross talk. 


\section{Discussion and Concluding remark}

We observed several impairments on WDM packet amplification caused by cross gain saturation. It appears not only as gain excursion but also additional noise come from inter-channel crosstalk. We have proposed to adopt EDF with larger erbium core for mitigation, previously. And we proved in this paper that our proposal is also effective in WDM enviroment.

There are three major features in our experimental result.

At first, reminding that there is no significant impairment with Type $\mathrm{B}$ and $\mathrm{C}$ in the condition of $\mathrm{LU}=33 \%$ while we observed serious gain excursion occured in $\mathrm{LU}=0.4 \%$, the change of link utilization according to the request of control plane induces the change of packet envelope which affect receiver condition. This dependency on link utilization is more serious than the gain excursion itself.

Secondly, the degree of impairment was cululative. It is not only the problem inside of independent OPS, but also the problem of optical packet network because the packets from different paths and hops should suffer different impairment.

Therefore, mitigation of excursion is important as same as enhancing the tolerance of receiver.

Thirdly, our observed excrusion occured within several hundred nano seconds and existing AGC cannot respond during packet. Fortunately, our mitigation inherently reduces the transient and it can be easily applied to conventional relatively low speed controlling method to supplement it. As far as the application of optical packet with short duration, it is desirable to adopt a EDF with larger active erbium core.

Further issues of our proposal are quantitatively investigated with modified formula.

Nonetheless, mitigation of gain transient of EDFA is one of most important issues not only for OPS but also for optical links, for whole optical packet transporting stratum.

\section{References}

1. Schulze, E., Malach, M., Raub, F.: All-Raman amplified links in comparison to EDFA links in case of switched traffic. $28^{\text {th }}$ European Conference on Optical Communication (2002) Symposium 3.8.

2. Sun, Y., Zyskind, J. L., Srivastava, A. K., Zhang, L.: Analytical formula for the transient response of erbium-doped fiber amplifiers. Applied optics 38, 9 (1999) 1682-1685

3. Nakaji, H., Nakai, Y., Shigematsu, M., Nishimura, M.: Superior high-speed automatic gain controlled erbium-doped fiber amplifiers. Optical fiber technology 9 (2003) 25-35

4. Zhao, C-L., Tam, H-Y., Guan, B-O., Dong, X., Wai, P. K. A., Dong, X.: Optical automatic gain control of EDFA using two oscillating lasers in a single feedback loop. Optics communications 225 (2003) 157-162

5. Awaji, Y., Furukawa, H., Wada, N., Chan, P., Man, R.: Mitigation of transient response of erbium-doped fiber amplifier for burst traffic of high speed optical packets. Submitted to Conference on Lasers and Electro-Optics (2007)

6. Saleh, A. A. M., Jopson, R. M., Evankow, J. D., Aspell, J.: Modeling of gain in erbiumdoped fiber amplifiers. IEEE Photon. Technol. Lett. 2, 10 (1990) 714-717 
7. Furukawa, H., Wada, N., Harai, H., Naruse, M., Otsuki, H., Katsumoto, M., Miyazaki, T., Ikezawa, K., Toyama, A., Itou, N., Shimizu, H., Fujinuma, H., Iiduka, H., Cincotti, G., Kitayama, K.: All-optical multiple-label-processing based optical packet swtich prototype and novel 1-Gb ethernet / $80(8 \lambda$ x 10) Gbps-wide colored optical packet converter with 8channel array burst-mode packet transceiver. Optical Fiber Communication Conference (OFC), OWC5 (2007)

8. Harai, H., Wada, N.: More than 1-Gbps photonic packet-switched networks using WDMbased packet compression. 8th OptoElectronics and Communications Conference (2003) 15E3-3 